\title{
83. Tetraploidy appeared in the Offspring of Triploid Ginbuna, Carassius auratus langsdorfii (Cyprinidae, Pisces)
}

\author{
By Akinori TakaI and Yoshio OJIMA \\ Department of Biology, Faculty of Science, \\ Kwansei Gakuin University, Nishinomiya 662 \\ (Communicated by Sajiro Makino, M. J. A., Dec. 12, 1983)
}

The ginbuna (Carassius auratus langsdorfii) is a member of the funa (C. auratus) having a wide distribution in Japan, with an inclination to the female in the sex ratio. In the Kanto district, the ginbuna so far obtained were all females having 3n-range (about 150) or 4n-range (about 200) chromosomes (Kobayashi et al., 1970). These $3 n$-individuals propagate unisexually by gynogenesis (Kobayashi, 1971). Also, the ginbuna from Lake Biwa were mostly female with 3n-range chromosomes produced by gynogenesis (Ojima and Asano, 1977). Recent investigations have revealed that $3 n$-individuals of the ginbuna distribute widely through Japan accompanied with bisexual diploid individuals of the ginbuna or the other subspecies (Kobayashi, 1978; Takai and Ojima, 1982). On the other hand, 4nindividuals were sometimes found only in the limited areas as the Kanto district. The present paper deals with the chromosomal and electrophoretical investigations of the tetraploid ginbuna, appeared in the offspring of the triploid ginbuna developed from the fertilized eggs treated by refrigeration after the artificial insemination with the sperm of the gengorobuna (C.a. cuvieri).

Materials and methods. The spawned eggs from a single specimen of the ginbuna were artificially inseminated with the sperm of a single gengorobuna. Fifteen minutes after the insemination, fertilized eggs were exposed to low temperature at $1^{\circ} \mathrm{C}$ for $15 \mathrm{~min}$. Ten individuals developing from the eggs thus treated, and the parental species, the ginbuna and the gengorobuna, were used for the present study. The chromosome preparations were made from cultured cells from fins (Ojima, 1978) as well as directly prepared kidney cells (Ojima and Takai, 1979) following the routine air-drying method with conventional stain. The BSG method by Sumner (1972) was employed for the C-banding analyses.

For electrophoretic analysis, specimens dissected for the chromosome studies were stored frozen at $-20^{\circ} \mathrm{C}$ until the use. Sampling of the muscle tissue and the horizontal starch-gel electrophoresis for 
muscle protein followed the methods after Ojima and Takai (1979). Electrophoresis for the non-specific esterase of the muscle, followed the hcrizontal acrylamide-gel method, was carried out at $20 \mathrm{~mA}$ for $6 \mathrm{~h}$ at $4{ }^{\circ} \mathrm{C}$ in $5 \%$ gel with $2 \mathrm{~mm}$ thickness and $20 \mathrm{~cm}$ width. BNbuffer (0.3 M Boric acid $+0.05 \mathrm{M} \mathrm{NaOH}, \mathrm{pH} 8.45)$ for electrode, and the mixed buffer of TC- $(0.2 \mathrm{M}$ Tris $+0.035 \mathrm{M}$ citric acid, $\mathrm{pH} 8.3)$ and BN-buffer $(3: 1)$ for gel, were used. After the end of run, the gel was stained for the non-specific esterase in the staining solution containing $1 \mathrm{ml}$ of $1 \% \quad \alpha$-naphthyl acetate, $50 \mathrm{mg}$ of Fast blue RR salt, and $50 \mathrm{ml}$ of $0.2 \mathrm{M}$ Tris buffer, $\mathrm{pH} 7.0$.

Results and discussion. The maternal ginbuna had 3n-range chromosomes with a modal number of 154 consisting of 84 metaand submetacentrics, 66 acrocentrics, and 4 minute elements, and the C-banding pattern was characterized by two larger submetacentrics with intensely stained short arms and four deeply stained minute elements. These results were identical with those of the

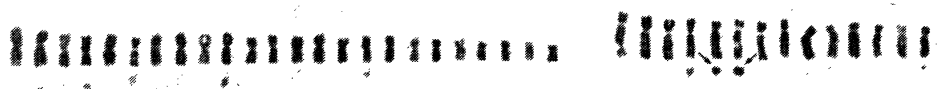

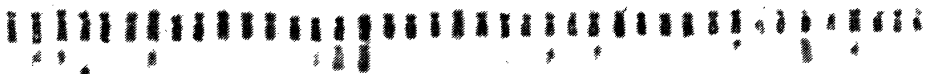

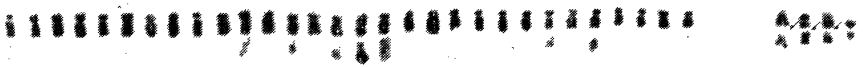

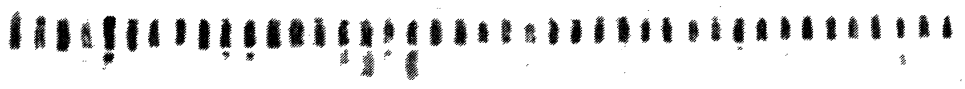

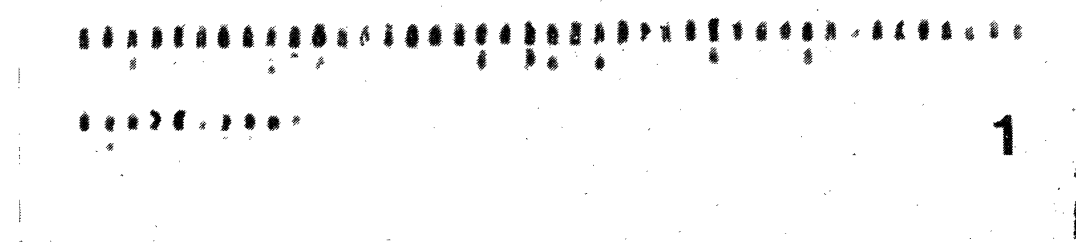

Fig. 1. The conventional stained karyotype and the C-banding pattern of the 4 n-offspring from the triploid ginbuna. Allows show the characteristic C-bands found in the short arms of two larger submetacentrics and in minute elements.

ginbuna reported by Ueda and Ojima (1978). The paternal gengorobuna had 100 diploid chromosomes consisting of 6 pairs of metacentrics, 18 pairs of submetacentrics, and 26 pairs of acrocentrics and they showed no characteristic C-banding pattern, as shown by Ojima et al. (1966), and Ueda and Ojima (1978). 
Ten offspring examined in this experiments were all females. Four from among ten offspring showed 4n-range chromosomes, having 204 in modal number, with 108 meta- and submetacentrics, 92 acrocentrics, and 4 minute elements (Fig. 1). These C-banding patterns were characterized by two larger submetacentrics with intense C-bands at the short arms, and four C-banded minute elements, as well as those of the maternal ginbuna, in spite of the difference of their chromosome number (Fig. 1). The other offspring, however, showed 3n-ranged chromosomes with the same karyotypic constitution and C-banding pattern as those of the maternal ginbuna. In comparison with the karyotype constitution and the C-banding pattern between the $4 \mathrm{n}$-offspring and their parents, it was clear that the 4n-offspring had a haploid chromosome set of the paternal gengorobuna and the triploid chromosome set of the maternal ginbuna.

In electrophoretic patterns of the muscle protein, the maternal ginbuna and the 3n-offspring showed the same pattern (Fig. 2-1) as a common pattern in the triploid ginbuna (Takai and Ojima, 1982). The $4 \mathrm{n}$-offspring showed a pattern similar to that of the maternal ginbuna, but the density of the $3 r d$ band in the 4 n-offspring was higher than that of the maternal ginbuna (Fig. 2-2). This 3rd band
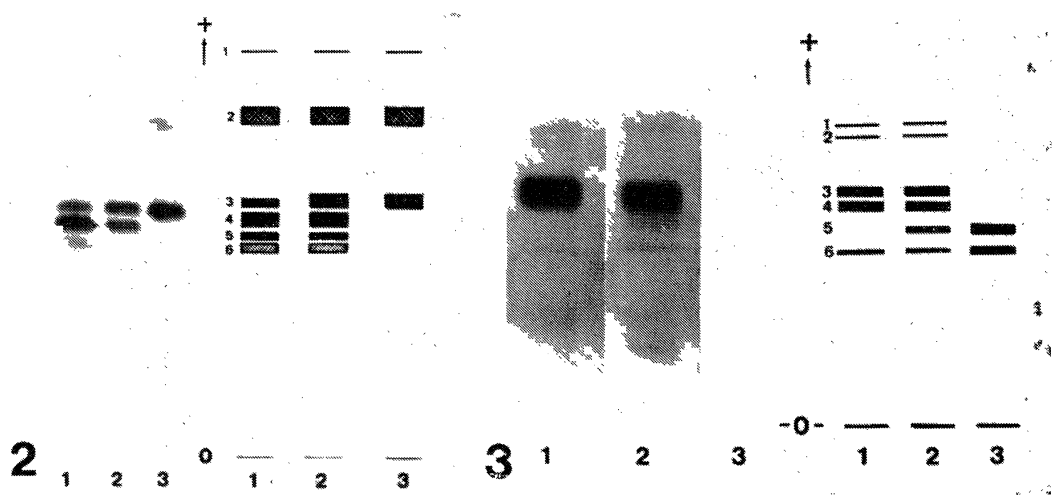

Figs. 2-3. 2: The electrophoretic patterns of the muscle protein. 2-1, the maternal ginbuna and the $3 n$-offspring; $2-2$, the 4 -offspring; $2-3$, the paternal gengorobuna. 3: The electrophoretic patterns of non-specific esterase of the muscle. $3-1$, the maternal ginbuna and the $3 n$-offspring; $3-2$, the $4 n$ offspring; 3-3, the paternal gengorobuna.

was characterized in the pattern of the paternal gengorobuna (Fig. 2-3). In non-specific esterase patterns of the muscle, the $3 n$-offspring had an identical pattern with that of the maternal ginbuna (Fig. 3-1). However, there was some difference in pattern of the maternal ginbuna and the 4 n-offspring. Obviously, the 5 th band in the $4 n$ - 
[Vol. 59(B),

offspring was not found in the maternal ginbuna (Fig. 3-2), but was characterized by the pattern of the paternal gengorobuna (Fig. 3-3). Thus, the electrophoretic patterns of the 4 -offspring apparently expressed hybrid specificities between the paternal and the maternal characters. These electrophoretical findings could support the suggestion from the chromosomal study as stated above.

Usually the gynogenetic form produces ova with non-reduced chromosomes and they develop by an activation of spermatozoa derived from the bisexual species, without the participation of the paternal genome in fertilization (Ojima and Asano, 1977). From the chromosomal and electrophoretical results it is evident that the 4 -offspring has a haploid genome derived from the paternal gengorobuna, in addition to a triploid genome of the maternal ginbuna. This fact indicates that the 4 n-offspring have been induced by the occurrence of unexpected fertilization by the union of the sperm nucleus and the egg nucleus through the gynogenetic reproduction, probably caused by the refrigeration of the fertilized eggs.

Thus, the present study revealed that tetraploidy was induced from the triploid ginbuna under the artificial condition. Such phenomenon would be possible to occur accidentally also under the natural condition. Kobayashi et al. (1977) suggested that the tetraploid form in the Kanto district was produced by gynogenesis. The artificial $4 \mathrm{n}$-individuals of our experiment were all females. So far as our findings were concerned, at least, the hybrid between the triploid ginbuna and the bisexual diploid funa as shown in this study would be an origin of the tetraploid ginbuna in nature.

Acknowledgement. We are grateful to Dr. Sajiro Makino, M. J.A., Professor Emeritus, Hokkaido University, for going over the manuscript with invaluable advice.

\section{References}

Kobayashi, H.: Zool. Mag. (Tokyo), 80, 316-322 (1971).

- Iden (The Heredity), 32(7), 28-38 (1978) (in Japanese).

Kobayashi, H., Kawashima, Y., and Takeuchi, N.: Japan J. Ichthyol., 17, 153-160 (1970).

Kobayashi, H., Nakano, K., and Nakamura, M.: Bull. Jpn. Soc. Sci. Fish., 43, 31-37 (1977).

Ojima, Y.: Proc. Japan Acad., 54B, 116-120 (1978).

Ojima, Y., and Asano, N.: ibid., 53B, 138-142 (1977).

Ojima, Y., and Takai, A.: ibid., 55B, 346-350 (1979).

Ojima, Y., Hitotsumachi, S., and Makino, S.: ibid., 42, 62-66 (1966).

Sumner, A. T.: Exp. Cell Res., 75, 304-306 (1972).

Takai, A., and Ojima, Y.: Proc. 53rd Ann. Meet. Zool. Soc. Japan., Zool. Mag., vol. 91, p. 610 (1982).

Ueda, T., and Ojima, Y.: Proc. Japan Acad., 54B, 283-288 (1978). 